\title{
Expansão das Fronteiras do Tratamento Percutâneo
}

\author{
Áurea J. Chaves
}

0 marcante sucesso das intervenções percutâneas em revascularizar pacientes com doença arterial coronária fez expandir para novos limites o tratamento de outras doenças cardíacas com a utilização de cateteres. A área da intervenção valvular, em especial, desenvolveu-se rapidamente nos últimos anos, passando a tratar de maneira eficiente e segura pacientes que até então tinham a cirurgia como única opção.

Este ano de 2008 foi marcado, entre outros avanços, pelos procedimentos de substituição percutânea da valva aórtica, que começam a ser realizados de forma rotineira nos centros de maior expressão científica da especialidade.

Esta edição da Revista Brasileira de Cardiologia Invasiva ( $\mathbf{R B C I}$ ) traz aos leitores a primeira publicação dos resultados do implante percutâneo de próteses valvulares aórticas realizado em instituição brasileira. O artigo de capa, de Sarmento-Leite et al., descreve a experiência inicial, realizada no Instituto de Cardiologia do Rio Grande do Sul, em Porto Alegre, RS, com o implante dessas próteses. Os autores apresentam, de forma didática, os critérios de seleção dos pacientes, a descrição do dispositivo, todas as etapas do procedimento e os resultados dessa nova técnica minimamente invasiva e com potencial para revolucionar o tratamento da doença valvar aórtica. Complementa a abordagem desse assunto o editorial de Grube e Buellesfeld, do HELIOS Heart Center de Siegburg, Alemanha. Esses autores, além de enfocar a exeqüibilidade, a segurança e a efetividade da intervenção em pacientes com estenose aórtica grave de alto risco cirúrgico, comentam sobre a necessidade da correta avaliação para identificar indivíduos anatomicamente adequados para essa técnica. Citam o crescimento exponencial dos procedimentos em todo o mundo nos últimos meses e antecipam em poucos anos a expansão da indicação para pacientes com atual recomendação cirúrgica clássica.

Esta edição especial de fim de ano traz, também, outros excelentes editoriais. Antonio Colombo e Azeem Latib, do San Raffaele Scientific Institute e EMO-GVM Centro Cuore Columbus, em Milão, Itália, abordam um dos desafios da intervenção coronária percutânea (ICP) na atualidade: o tratamento das lesões em bifurcação. Lembram a complexida- de do procedimento e comentam aspectos da eficácia e da segurança dos stents farmacológicos nesse cenário. Dissecam os resultados do artigo de Mendes et al. e os comparam aos resultados dos estudos SCANDESTENT, Nordic e ao recentemente apresentado CACTUS. Opinam que a estratégia do provisional stent com stents farmacológicos deve ser o padrão de indicação na atualidade para o tratamento de bifurcações que não envolvam o tronco da artéria coronária esquerda.

Eugenia Nikolsky e Roxana Mehran, do Columbia University Medical Center e da Cardiovascular Research Foundation, de Nova York, Estados Unidos, exploram o tópico da falência renal aguda em pacientes com infarto agudo do miocárdio tratados com ICP primária. Comentam a respeito da insuficiência renal crônica, que vem sendo reconhecida como um dos mais fortes fatores que influenciam a mortalidade em pacientes submetidos a ICP. Discutem os achados de Passos et al., em particular a necessidade da rápida estabilização das instabilidades respiratória e hemodinâmica dos pacientes tratados com ICP primária, para minimizar a deterioração da função renal. Lembram também da logística particular da ICP primária, que impede a hidratação pré-procedimento adequada, e recomendam esforços para a redução do volume dos meios de contraste nesses casos.

Carísi Polanczyk, do Hospital de Clínicas de Porto Alegre, RS, ao discutir o artigo de Ghem et al., recorda avanços recentes na identificação de novos fatores que dão informações prognósticas e guiam condutas terapêuticas na doença aterosclerótica coronária. Faz revisão do surpreendente papel que a bilirrubina parece ter, como agente antioxidante e protetor para a aterogênese, e adverte para a necessidade do muito a ser esclarecido em relação aos mecanismos moleculares e celulares relacionados à principal causa de morte em nosso País.

Por fim, Valmir Fontes et al., do Instituto Dante Pazzanese de Cardiologia, de São Paulo, SP, um dos ícones brasileiros das intervenções percutâneas em cardiopatias congênitas, fazem completa revisão histórica do fechamento percutâneo das comunicações interatriais (CIAs) e opinam a respeito da nova prótese utilizada no fechamento de CIA ostium primum descrita no relato de caso de Chamié et al. 
No encerramento deste produtivo ano de 2008, agradeço aos autores, revisores, componentes do Corpo Editorial da $\mathbf{R B C I}$, funcionários, diretores e, em especial, ao presidente da Sociedade Brasileira de Hemodinâmica e Cardiologia Intervencionista, Luiz Alberto
Mattos, desejando a todos Feliz Natal e que 2009 seja ainda melhor!

Áurea J. Chaves

Editora 